\title{
Prevalência de sintomas da doença do refluxo gastroesofágico em crianças com sobrepeso e obesidade, de Salvador, Bahia
}

\author{
Prevalence of Gastroesophageal Reflux disease in children with obesity in Salvador,Bahia
}

\begin{abstract}
Sabrina Carneiro Rocha ${ }^{1}$, Rita Franca ${ }^{2}$, Luciana Rodrigues Silva ${ }^{3}$, Cibele Dantas Ferreira Marques ${ }^{4}$
${ }^{1}$ Médica Residente de Gastroenterologia e Hepatologia Pediátricas da Universidade Federal da Bahia-UFBA;

${ }^{2}$ Médica Preceptora Doutora do Serviço de Gastroenterologia e Hepatologia Pediátricas da UFBA; ${ }^{3}$ Professora Titular Doutora e Chefe do Serviço de Gastroenterologia e Hepatologia Pediátricas da UFBA; ${ }^{4}$ Médica Professora, Mestre, Preceptora Serviço de Gastroenterologia e Hepatologia Pediátricas da UFBA.
\end{abstract}

\begin{abstract}
Resumo
Introdução: A obesidade é uma doença que vem aumentando de forma alarmante em todas as idades e classes sociais de todo o mundo e que atinge adultos e crianças. Na infância tem associação comprovada com várias patologias e mais recentemente estudos foram realizados para correlacionar obesidade com a presença de sintomas gastrointestinais, dentre eles a doença do refluxo gastroesofágico. Objetivo: Determinar a prevalência dos sintomas da doença do refluxo gastroesofágico em crianças com sobrepeso e obesas, de Salvador-Bahia. Método: Estudo descritivo de prevalência, onde foram selecionados 77 pacientes entre 6 a 17 anos com diagnóstico de sobrepeso e obesidade atendidos no Serviço de Gastroenterologia e Hepatologia Pediátricasda Universidade Federal da Bahia. Após classificação nutricional, os pacientes responderam a um questionário, enfatizando-se achados relacionados com sintomas de refluxo. Em cada paciente foi aplicado um escore para o cálculo da prevalência, em que foram considerados positivos para a doença do refluxo gastroesofágico, a presença de três ou mais destes sintomas. Resultados: Participaram do estudo 77 pacientes com idade de 6 a 17 anos com diagnóstico de sobrepeso e obesidade. Os sintomas mais prevalentes, foram soluços e dor abdominal em 43 pacientes $(55,9 \%)$ e em 35 pacientes $(45,5 \%)$ respectivamente. Regurgitações e vômitos foram os sintomas menos prevalentes em 18 pacientes $(23,4 \%)$ e 13 pacientes $(16,9 \%)$ respectivamente. A prevalência geral nesta população de sintomas do refluxo gastroesofágico foi de 14,2\%. Conclusão: Foi observada uma correlação positiva entre a obesidade infantil e a presença de sintomas da doença do refluxo gastroesofágico.
\end{abstract}

Palavras chave: Obesidade. Criança. Refluxo Gastroesofágico . Pediatria.

\section{Abstract}

Introduction: Obesity is a disease that is increasing alarmingly in all ages and social classes around the world, and that affects adults and children. In childhood is associated with various pathologies and confirmed more recently studies have been conducted in order to prove their correlation with the presence of gastrointestinal symptoms, including gastroesophageal reflux disease. Objective: To determine the prevalence of symptoms of gastroesophageal reflux disease in overweight and obese, of Salvador, Bahia. Methodology: A descriptive study of prevalence, where 77 patients were selected from 6 to 17 years with a diagnosis of overweight and obesity in the obesity clinic attended the Teacher Center for Pediatric Hozaná olive (CPPHO). After nutritional classification, underwent a detailed questionnaire, with emphasis on findings related to gastroesophageal reflux symptoms such as vomiting, hiccups, regurgitation, choking, heartburn, abdominal pain and hoarseness. In each patient a score was applied to calculate the prevalence, they were considered positive for gastroesophageal reflux disease, the presence of three or more of sintomas. Resultados: the study involved 77 patients aged 6-17 years with a diagnosis of overweight and obesity. The most prevalent symptoms were sobs and abdominal pain with 43 patients $(55.9 \%)$ and 35 patients $(45.5 \%)$ respectively. Regurgitation and vomiting symptoms were less prevalent, with 18 patients $(23.4 \%)$ and 13 patients $(16.9 \%)$ respectively. The prevalence of gastroesophageal reflux symptoms was $14.2 \%$. Conclusion: We observed a positive correlation between childhood obesity and the presence of symptoms of gastroesophageal reflux disease.

Keywords: Obesity. Child. Gastroesophageal Reflux. Pediatrics.

\section{INTRODUÇÃO}

A obesidade é uma doença que vem aumentando de forma alarmante em todas as idades e classes sociais de todo o mundo. [6] 0 rápido aumento da obesidade infantil tem se tornado um dos maiores problemas enfrentados pelos pediatras. [7] Há a estimativa de 22 milhões de crianças com sobrepeso no mundo. [8]

Recebido em 25/12/2011; revisado em 06/02/2012.

Correspondência / Correspondence: Luciana Rodrigues Silva. Centro Pediátrico Professor Hosannah de Oliveira - UFBA. Rua Pe. Feijó, s/n - Canela. 40110-70 Salvador - BA - Brasil. Tel.: (71) 33396134. Email: lupe.ssa@uol.com.br
Segundo a Organização Mundial de Saúde, a prevalência da obesidade infantil tem crescido em torno de 10 a $40 \%$ na maioria dos países europeus nos últimos 10 anos, assim como nos países em desenvolvimento. [6]

A obesidade na infância tem associação comprovada com diversas outras patologias como doenças cardiovasculares, diabetes, hipertensão, hiperlipidemia, esteatohepatite, síndrome metabólica, problemas psicológicos, alterações ortopédicas, dentre outros. Têm sido realizados estudos com o intuito de comprovar a correlação da obesidade com a presença 
de sintomas gastrointestinais, dentre eles a doença do refluxo gastroesofágico. [7]

Assim como a obesidade, o refluxo gastroesofágico é uma patologia muito comum, com patogênese multifatorial, que afeta mais de $40 \%$ da população. A relação entre estas duas doenças tem sido relatada por prevalência e causalidade, já que a obesidade tem sido apontada como um grande fator de risco para o desenvolvimento de refluxo gastroesofágico, e suas complicações, incluindo o esôfago de Barret e o adenocarcinoma de esôfago. [9]

A principal anormalidade fisiopatológica na doença do refluxo gastroesofágico envolve a disfunção do esfíncter esofageano inferior do esôfago. Estudos já demonstraram que a pressão basal do esfíncter esofageano inferior do esôfago é semelhante entre obesos e não obesos, entretanto os obesos apresentam maior prevalência de hérnia de hiato, além desta alteração ser maior nestes pacientes. A obesidade também está relacionada com o aumento do relaxamento transitório do esfíncter esofageano inferior, refluxo pós prandial e exposição ácida do esôfago. A frequência elevada deste relaxamento transitório está proporcionalmente correlacionada com o aumento do índice de massa corpórea e circunferência da cintura. O perfil de pressão ao longo da junção esôfago gástrica também é alterado em pacientes obesos, o que aumenta, consequentemente, o risco de refluxo gastroesofágico. O aumento da circunferência da cintura parece ser o principal fator envolvido com o aumento da pressão intra abdominal e predisposição ao refluxo gastroesofágico. Além destes fatores, os pacientes obesos normalmente fazem refeições em grande quantidade e com alto valor calórico, o que está relacionado com um esvaziamento gástrico lento e aumento da freqüência do relaxamento transitório do esfíncter esofagiano inferior. [9]

Em adultos, a associação entre obesidade e doença do refluxo gastroesofágico é bem estabelecida, porém dados semelhantes na literatura pediátrica são escassos. O primeiro estudo publicado que identificou essa relação de causalidade entre o aumento do índice de massa corpórea e o refluxo gastroesofágico foi realizado na Noruega por Stordal et al. em 2006 envolvendo crianças de 7 a 16 anos, relatado por sintomas ou alterações na pHmetria [10]. Pashankar et al em 2009 realizou estudo com crianças de 7 a 16 anos de New Haven, e observou uma prevalência de 13,1\% de sintomas do refluxo gastroesofágico em crianças obesas, valor muito acima dos $2 \%$ que se observa nas crianças não obesa. Essa mesma prevalência chegou a $20 \%$ quando levado em consideração apenas as crianças com obesidade grave. [7] Por outro lado, Patel et al em 2010 publicou um estudo que envolveu 230 crianças de Nova York, com idade entre 2 e 20 anos, com sintomas de da doença do refluxo gastro esofágico, onde a obesidade foi observada em $29,1 \%$ dos pacientes.
Todos os pacientes realizaram endoscopia digestiva alta e a prevalência da doença do refluxo gastroesofágico foi de $23,9 \%$, com valor semelhante de $24,5 \%$ encontrado nas crianças não obesas. [11]

\section{OBJETIVO}

Determinar a prevalência dos sintomas da doença do refluxo gastroesofágico em crianças com sobrepeso e obesidade.

\section{MÉTODOS}

Trata-se de um estudo descritivo de prevalência, onde foram selecionados 77 pacientes escolares e adolescentes entre 6 a 17 anos com diagnóstico de sobrepeso e obesidade de Salvador Bahia. Todos os pacientes foram acompanhados no Serviço de Gastroenterologia e Hepatologia Pediátricas da UFBA, juntamente com acompanhamento nutricional e psicológico específico.

Utilizou-se o critério de curva de percentil de IMC para classificação nutricional das crianças. Foi definido sobrepeso como o índice de massa corporal (IMC) maior ou igual ao percentil 85 e menor que 95, e para a obesidade o IMC maior ou igual a 95.

Inicialmente procedeu-se a classificação nutricional e em seguida os pacientes com obesidade ou sobrepeso foram submetidos a um questionário detalhado, enfatizando-se achados relacionados com sintomas de refluxo gastroesofágico como vômitos, soluços, regurgitação, engasgos, azia, rouquidão e dor abdominal. Em cada paciente foi aplicado um escore de sintomas para o cálculo da prevalência dos sintomas da doença do refluxo gastroesofágico, em que foram considerados positivos para a doença do refluxo gastroesofágico, a presença de três ou mais destes sintomas. O escore de sintomas para o diagnóstico da doença do refluxo foi usado inicialmente e validado em estudo prévio realizado por Stordal et al em 2006, em que o escore positivo com a presença de três ou mais dos sintomas selecionados que sugerem o diagnóstico da doença do refluxo gastroesofágico, utilizando-se a phmetria de 24 horas como método de confirmação. Apesar de não ser o método ideal para o diagnóstico da doença do refluxo gastroesofágico, este escore foi validado para a sua utilização em estudos epidemiológicos. [7]

Todos os pacientes foram acompanhados regularmente no referido Ambulatório.

A análise dos resultados foi realizada através do software estatístico SPSS, versão 17.0 Windows, utilizando-se também medidas de frequência.

O paciente e/ou seu representante legal foi esclarecido sobre o estudo e assinou o Termo de Consentimento livre e esclarecido. De acordo com as normas propostas para pesquisas envolvendo seres humanos, o protocolo do estudo foi aprovado pelo Comitê de Ética e Pesquisa do HUPES. 


\section{RESULTADOS}

Um total de 77 pacientes com idade entre 6 a 17 anos e com o diagnóstico de sobrepeso e obesidade participaram do estudo. Cinquenta pacientes foram do sexo feminino $(64,9 \%)$ e 27 do sexo masculino $(35,1 \%)$. A obesidade foi observada em 47 pacientes (61\%).

A prevalência isolada de cada sintoma encontrado nos pacientes foi variável, sendo que soluços e dor abdominal foram os sintomas mais prevalentes, com 43 pacientes $(55,9 \%)$ e 35 pacientes $(45,5 \%)$ respectivamente. A rouquidão foi relatada por 26 pacientes $(33,8 \%)$, a pirose em 24 pacientes $(31,2 \%)$ e os engasgos em 23 pacientes (29,9\%). As regurgitações e os vômitos foram os sintomas menos prevalentes, com 18 pacientes $(23,4 \%)$ e 13 pacientes $(16,9 \%)$ respectivamente. Após coletados esses dados, a prevalência dos sintomas da doença do refluxo gastroesofágico foi obtida com a aplicação do escore de sintomas. A prevalência dos sintomas da doença do refluxo gastroesofágico foi de $14,2 \%$, já que 11 pacientes apresentaram três ou mais dos sintomas abordados (Tabela 1).

Tabela 1 - Prevalência dos sintomas do refluxo gastroesofágico em crianças obesas, Salvador, Bahia

\begin{tabular}{lc}
\hline Sintomas & Prevalência \\
\hline Soluços & $55,9 \%$ \\
Dor abdominal & $45,5 \%$ \\
Rouquidão & $33,8 \%$ \\
Pirose & $31,2 \%$ \\
Engasgos & $29,9 \%$ \\
Regurgitação & $23,4 \%$ \\
Vômitos & $16,9 \%$ \\
Prevalência total & $14,2 \%$ \\
(segundo escore empregado) & \\
\hline
\end{tabular}

\section{DISCUSSÃO}

A prevalência da obesidade tem crescido muito nas crianças e a presença de sintomas de refluxo gastroesofágico tem se incorporado na lista dos problemas associados com a obesidade infantil. [1]

O presente estudo observou uma elevada prevalência de sintomas do refluxo gastroesofágico $(14,2 \%)$, tanto isoladamente quanto sob a forma de escore de sintomas para o diagnóstico desta doença. 0 sintoma mais frequente foi o soluço $(55,9 \%)$, seguido de dor abdominal $(45,5 \%)$ e pirose $(31,2 \%)$, que são sintomas mais comuns em crianças maiores e de acordo com a faixa etária das crianças que participaram do estudo. Os sintomas menos prevalentes foram regurgitação $(23,4 \%)$ e vômitos $(16,9 \%)$, provavelmente também pela idade dos pacientes, já que estes últimos sintomas tendem a ser mais exuberantes em crianças menores.
A prevalência de sintomas do refluxo gastroesofágico em crianças obesas encontrado neste estudo $(14,2 \%)$ foi semelhante aos resultados encontrados por Stordal et al $(16,2 \%)$ e por Pashankar et al $(13,1 \%)$. [1] que usaram metodologia semelhante. Esses dados sugerem que existe uma relação positiva entre a obesidade infantil e o risco de desenvolver a doença do refluxo gastroesofágico, por altreações de maior depósito de gordura no abdomen e alterações outras digestórias morfológicas e funcionais, relacionadas com a permeamilidade, motilidade, sistema endócrino, flora intestinal e outros fatores. Por outro lado, existem outros estudos como o de Patel et al (D) que não observaram esta correlação. É consenso que para melhora da Doença do Refluxo gastroesofágico, estes pacientes tem uma das medidas terapêuticas representada pela necessidade absoluta de perda de peso.

\section{CONCLUSÃO}

O presente estudo observou a presença de sintomas da doença do refluxo gastroesofágico em pacientes com sobrepeso e obesidade. Avaliou-se apenas os aspectos clínicos, sem complementação com exames laboratoriais, o que representa numa limitação deste trabalho. É necessário a realização de novos estudos prospectivos que avaliem os aspectos clínicos, mas também os exames complementares como a phmetria de 24 horas e a endoscopia digestiva alta simultaneamente, para avaliar com melhor precisão essa associação entre a obesidade infantil e a doença do refluxo gastroesofágico com populações de diferentes áreas geográficas.

\section{REFERÊNCIAS}

1-OLIVEIRA, AM. Triglycerides and alanine aminotransferase as screening markers for suspected fatty liver disease in obese children and adolescents. Horm. Res., Basel, v. 71, n. 2, p. 83-88, 2009.

2-HOSSAIN, P; KAWAR, B; EL NAHAS, M. Obesity and diabetes in the developing world - a growing challenge. N. Engl. J. Med., Boston, v. 356, n. 3, p. 213-215, 2007.

3 - BICCAS, BN. et al. Maior prevalência de obesidade na doença do refluxo gastroesofágico erosiva. Arq. Gastroenterol.,São Paulo, v. 46 , n. 1, p. 15-19, jan/mar 2009.

4 - POPKIN, Barry M. The nutrition transition and obesity in the developing world. J. Nutr., Philadelphia, v. 131, n. 3, p. 871S-873S, 2001.

5 - OBESITY: preventing and managing the global epidemic. Report of a WHO consultation. World Health Organ. Tech. Rep. Ser., Geneva, v.894, n.1-12, p. 1-25, 2000.

6 - MELLO, ED; LUFT, VC; MEYER, F. Obesidade infantil: como podemos ser eficazes?. J. Pediatr., Rio de Janeiro, v. 80, n. 3, p 173-82, 2004.

7 -PASHANKAR, DS. et al. Increased Prevalence of Gastroesophageal Reflux Symptoms in Obese Children evaluated in an Academic Medical Center. J. Clin. Gastroenterol., New York, v. 43, n. 5 , p. 410-413, 2009. 
8- Scottish Intercollegiate Guidelines Network - Manegement of obesity in children and young people. Royal college of physicians. Disponivel em: < http:// www.sign.ac.uk>. Acessado: 19 de abril 2005.

9 - ANAND, Girish, KATZ, Philip O. Gastroesophageal Reflux Disease and Obesity. Gastroenterol. Clin. North Am. Philadelphia, v. 39, n. 1, p. 39-46, 2010.
10 - STORDAL, K. et al. Asthma and overweight are associated with symptoms of gastro-oesophageal reflux. Acta Paediatr., Stockholm, v. 95, n. 10, p. 1197-1201, 2006.

11 - PATEL, NR. et al. The Association between Chilhood Overweight and Reflux Esophagitis. J. Obes., New York, v. 2010, pii. 136909, 2010. 